\title{
The downregulation of SnoN expression in human renal proximal tubule epithelial cells under high-glucose conditions is mediated by an increase in Smurf2 expression through TGF- $\beta 1$ signaling
}

\author{
XIUJI LI ${ }^{1,3}$, ZONGLI DIAO $^{1}$, JIAXIANG DING ${ }^{1}$, RUIXIA LIU ${ }^{2}$, LIYAN WANG $^{1}$, WEN HUANG $^{3}$ and WENHU LIU ${ }^{1}$ \\ ${ }^{1}$ Department of Nephrology, Beijing Friendship Hospital, Faculty of Kidney Diseases, Capital Medical University; \\ ${ }^{2}$ Department of Infectious Diseases, Beijing Friendship Hospital, Capital Medical University; ${ }^{3}$ Department of Nephrology, \\ Beijing Tongren Hospital, Faculty of Kidney Diseases, Capital Medical University, Beijing, P.R. China
}

Received June 16, 2015; Accepted December 18, 2015

DOI: $10.3892 /$ ijmm.2015.2448

\begin{abstract}
Transforming growth factor (TGF)- $\beta 1$ is a profibrotic cytokine that plays a critical role in the progression of diabetic nephropathy (DN). Previous studies have demonstrated that the Smad transcriptional co-repressor, Ski-related novel protein $\mathrm{N}$ (SnoN), an antagonizer of TGF- $\beta 1 /$ Smad signaling, is downregulated in the kidneys of diabetic rats; however, the underlying molecular mechanisms remain elusive. In the present study, we demonstrated that the upregulation of Smad ubiquitination regulatory factor-2 (Smurf2), through TGF- $\beta 1 /$ Smad signaling, contributes to the downregulation of SnoN under high-glucose conditions in primary human renal proximal tubule epithelial cells (hRPTECs). The hRPTECs were cultured in high-glucose (30 mmol/l D-glucose) medium in the presence or absence of either the proteasome inhibitor, MG132, or the TGF- $\beta$ type I receptor kinase inhibitor, SB-431542. Small interfering RNA (siRNA) was used to silence Smurf2. The expression levels of SnoN, Smurf2, Smad2 and phosphorylated (p-)Smad 2 were measured by western blot analysis and RT-qPCR. The protein levels of SnoN were markedly downregulated, while its mRNA levels were increased in the hRPTECs cultured under high-glucose conditions. The protein and mRNA levels of Smurf 2 were significantly increased under high-glucose conditions. The knockdown of Smurf2 increased SnoN expression in the hRPTECs cultured in high-glucose medium. Moreover, MG132 partially inhibited SnoN degradation in the hRPTECs under high-glucose conditions and SB-431542 decreased the phosphorylation of Smad2 and the expression of Smurf2 induced under high-glucose conditions.
\end{abstract}

Correspondence to: Professor Wenhu Liu or Dr Zongli Diao, Department of Nephrology, Beijing Friendship Hospital, Faculty of Kidney Diseases, Capital Medical University, 95 Yong-An Road, Beijing 100050, P.R. China

E-mail: liuwenhu2014@126.com

E-mail: diaoted@163.com

Key words: transforming growth factor- $\beta 1$, SnoN, Smad ubiquitination regulatory factor-2, diabetic nephropathy, $\mathrm{Smad} 2$
Taken together, the findings of this study demonstrate that the downregulation of SnoN expression in hRPTECs under highglucose conditions is mediated by the increased expression of Smurf 2 through the TGF- $\beta 1 /$ Smad signaling pathway.

\section{Introduction}

Diabetic nephropathy (DN) is a major microvascular complication of diabetes mellitus (DM) that invariably leads to end-stage renal disease (ESRD). Although DN was traditionally considered a primarily glomerular disease, accumulating evidence indicates that renal tubules play an important role in the pathogenesis of DN (1-3). However, the mechanisms through which the deregulation of renal tubules contributes to the development of DN remain largely unknown.

Transforming growth factor (TGF)- $\beta 1$ initiates intracellular signaling by binding and activating transmembrane type I and II serine/threonine kinase receptors, which in turn activate the downstream mediators, Smad2 and Smad3. Activated Smad 2 and Smad3 undergo phosphorylation and heteroligomerize with Smad4 to form the Smad complex, which then translocates to the nucleus to regulate the transcription of TGF- $\beta 1$ target genes $(4,5)$. Under normal physiological conditions, TGF- $\beta 1 /$ Smad signaling is tightly controlled by a negative regulatory mechanism. Ski-related novel protein N (SnoN) is a Smad transcriptional co-repressor $(6,7)$ that negatively regulates TGF- $\beta 1 /$ Smad signaling by binding and repressing Smad complexes to activate gene transcription $(8,9)$. Thus, the abundance and activity of SnoN in a given circumstance may determine the final response of cells to TGF- $\beta 1$ stimulation. TGF- $\beta 1$ is a profibrotic cytokine that has been shown to play a key role in the pathophysiology of $\mathrm{DN}$, in both experimental models of DN and in patients (4). High glucose activates TGF- $\beta 1$ signaling, which in turn stimulates tubule epithelial cells to overproduce extracellular matrix (10). A large body of evidence has indicated that TGF- $\beta 1$ plays a critical role in the development of tubulointerstitial fibrosis in DN $(4,10-12)$. However, the molecular mechanisms underlying the role of TGF- $\beta 1$ in the pathogenesis of DN remain elusive.

In our previous study, we demonstrated that $\mathrm{SnoN}$ protein was downregulated progressively in the kidneys of diabetic 
rats and primary proximal tubule epithelial cells under highglucose conditions (13). Moreover, we demonstrated that SnoN inhibited high-glucose-induced epithelial-mesenchymal transition (EMT) in renal tubule cells (13). These data suggest that the loss of SnoN may lead to tubulointerstitial damage in DN through uncontrolled TGF- $\beta 1 /$ Smad signaling. However, the regulatory mechanisms responsible for the decrease in SnoN expression under high-glucose conditions remain to be elucidated. Of note, Smad ubiquitination regulatory factor-2 (Smurf2), an E3 ubiquitin ligase, has been shown to be increased in renal fibrosis induced by obstructive injury and to promote the downregulation of SnoN $(14,15)$. Considering that TGF- $\beta 1$ signaling is commonly upregulated in obstructive nephropathy and DN, we hypothesized that the upregulation of Smurf 2 through TGF- $\beta 1 /$ Smad signaling may contribute to the downregulation of SnoN in DN.

In this study, we examined the expression of SnoN in human renal proximal tubule epithelial cells (hRPTECs) cultured in high-glucose (30 mmol/l D-glucose) medium in the presence or absence of either the proteasome inhibitor, MG132, or the TGF- $\beta$ type I receptor inhibitor, SB-431542. We further determined the protein levels of SnoN following the silencing of Smurf 2 by small interfering RNA (siRNA) in the hRPTECs under high-glucose conditions.

\section{Materials and methods}

Cell culture. The hRPTECs were purchased from ScienCell Research Laboratories (San Diego, CA, USA). The cells were cultured in fibronectin-coated flasks using epithelial cell medium (ScienCell Research Laboratories) supplemented with growth additives, $2 \%$ fetal bovine serum (FBS) and penicillin/streptomycin. The cells were cultured at $37^{\circ} \mathrm{C}$ in a humidified incubator in the presence of $5 \% \mathrm{CO}_{2}$. Actively proliferating hRPTECs at the third passage were used in the subsequent experiments.

Cell treatments. When the cells reached $60-80 \%$ confluence, the culture medium was replaced with serum-free epithelial cell medium and the cells were starved for $16 \mathrm{~h}$ to synchronize cell growth. The cells were incubated in media containing various concentrations of glucose: normal glucose $(5.5 \mathrm{mmol} / \mathrm{l}$ D-glucose), high glucose (30 mmol/1 D-glucose) or in medium of a high osmolarity $(5.5 \mathrm{mmol} / 1 \mathrm{D}$-glucose $+24.5 \mathrm{mmol} / \mathrm{l}$ D-mannitol). The cells were incubated for 2, 12, 24, 48 and 72 h, and collected at various time points for RNA and protein extraction. D-glucose and D-mannitol were purchased from Sigma-Aldrich (St. Louis, MO, USA).

For treatment with MG132 (proteasome inhibitor), following starvation for $16 \mathrm{~h}$, the cells were treated with $1.0 \mu \mathrm{mol} / 1 \mathrm{MG} 132$ (Selleck Chemicals, Houston, TX, USA) for $48 \mathrm{~h}$ in media containing $5.5 \mathrm{mmol} / 1 \mathrm{D}$-glucose or $30 \mathrm{mmol} / \mathrm{l} \mathrm{D}$-glucose. For treatment with SB-431542 (TGF- $\beta$ type I receptor inhibitor), following starvation for $16 \mathrm{~h}$, the cells were treated with various concentrations of SB-431542 (Sigma-Aldrich) or the vehicle $(0.1 \% \mathrm{DMSO})$ for $48 \mathrm{~h}$ in medium containing $30 \mathrm{mmol} / \mathrm{l} \mathrm{D}$-glucose.

Gene silencing by siRNA. To facilitate the optimization of lipid-mediated transfection for RNA interference (RNAi) experiments, BLOCK-iT ${ }^{\mathrm{TM}}$ Alexa Fluor ${ }^{\circledR}$ Red Fluorescent Control (Invitrogen, Carlsbad, CA, USA), an Alexa Fluor ${ }^{\circledR} 555$ labeled, double-stranded RNA (dsRNA) duplex were transfected into the hRPTECs according to the manufacturer's instructions. The transfection efficiency was roughly determined by calculating the ratio of red fluorescence cells among the total cells. Subsequently, the hRPTECs were transiently transfected with negative control siRNA (Cat. no. 12935200), Smurf2 siRNA-1 (ID\#VHS41440), or Smurf2 siRNA-2 (ID\#VHS41441; Invitrogen) using Lipofectamine RNAiMAX reagent according to the manufacturer's instructions (Invitrogen). Briefly, the cells were plated into 6-well plates in medium without antibiotics and grown to 50-60\% confluence at the time of transfection. The siRNAs and Lipofectamine RNAiMAX reagent were diluted in Opti-MEM I reduced serum medium (Invitrogen), separately. The diluted siRNA was then added to the diluted Lipofectamine RNAiMAX reagent and incubated at room temperature for $5 \mathrm{~min}$. The final concentration of siRNA in the medium was $20 \mathrm{nM}$. The cells were incubated in serumfree medium with siRNA-lipid complex for $6 \mathrm{~h}$ at $37^{\circ} \mathrm{C}$. The medium was then replaced with medium with or without $30 \mathrm{mmol} / \mathrm{l} \mathrm{D}$-glucose, and the cells were incubated for an additional $48 \mathrm{~h}$. The control cells refer to the untransfected cells cultured for $48 \mathrm{~h}$ cultured under high-glucose conditions. The mock-transfected cells refer to the cells cultured for $48 \mathrm{~h}$ in media containing high glucose and diluted transfection reagent.

Western blot analysis. Total protein from the cultured cells was extracted using RIPA lysis buffer containing phosphatase inhibitors and protease inhibitors (Roche, Mannheim, Germany). Protein concentrations were determined using the bicinchoninic acid (BCA) assay. Equal amounts of proteins were separated by $10 \%$ sodium dodecyl sulfate-polyacrylamide gel electrophoresis (SDS-PAGE) and then transferred onto polyvinylidene fluoride (PVDF) membranes (Millipore Corp., Bedford, MA, USA). The membranes were blocked in 5\% non-fat milk for $1 \mathrm{~h}$ and incubated with primary antibody at a dilution recommended by the manufacturer overnight at $4^{\circ} \mathrm{C}$. The primary antibodies used were as follows: anti-Smad2 (ab40855), antiphospho-Smad2 (ab53100; both from Abcam, Cambridge, MA, USA), anti-Smurf2 (sc-25511), anti-SnoN (sc-9141), anti-SnoN (sc-9595) and anti- $\beta$-actin (sc-130656; all from Santa Cruz Biotechnology, Inc., Santa Cruz, CA, USA). After washing with TBST (10 mM Tris, $\mathrm{pH} 8.0,150 \mathrm{mM} \mathrm{NaCl}$, and $0.1 \%$ Tween-20), the membranes were incubated for $1 \mathrm{~h}$ with horseradish peroxidase-conjugated secondary antibody (Cell Signaling Technology, Inc., Boston, MA, USA) at a dilution of 1,3000. The membranes were processed using an enhanced chemiluminescence kit (Millipore Corp.) and the images were captured using the ChemiDoc XRS+ imaging system (Bio-Rad Laboratories, Inc., Hercules, CA, USA). $\beta$-actin was used as a loading control. Quantitative analysis of the western blot analysis data was performed by measuring the intensity of the band signals using Image Lab 3.0 software (Bio-Rad Laboratories, Inc.).

Reverse transcription-quantitative polymerase chain reaction $(R T-q P C R)$. Total RNA was isolated with TRIzol reagent (Invitrogen) according to the manufacturer's instructions. RNA concentration and quality were determined by 

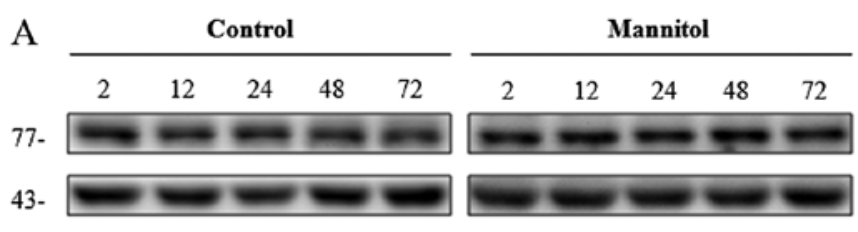
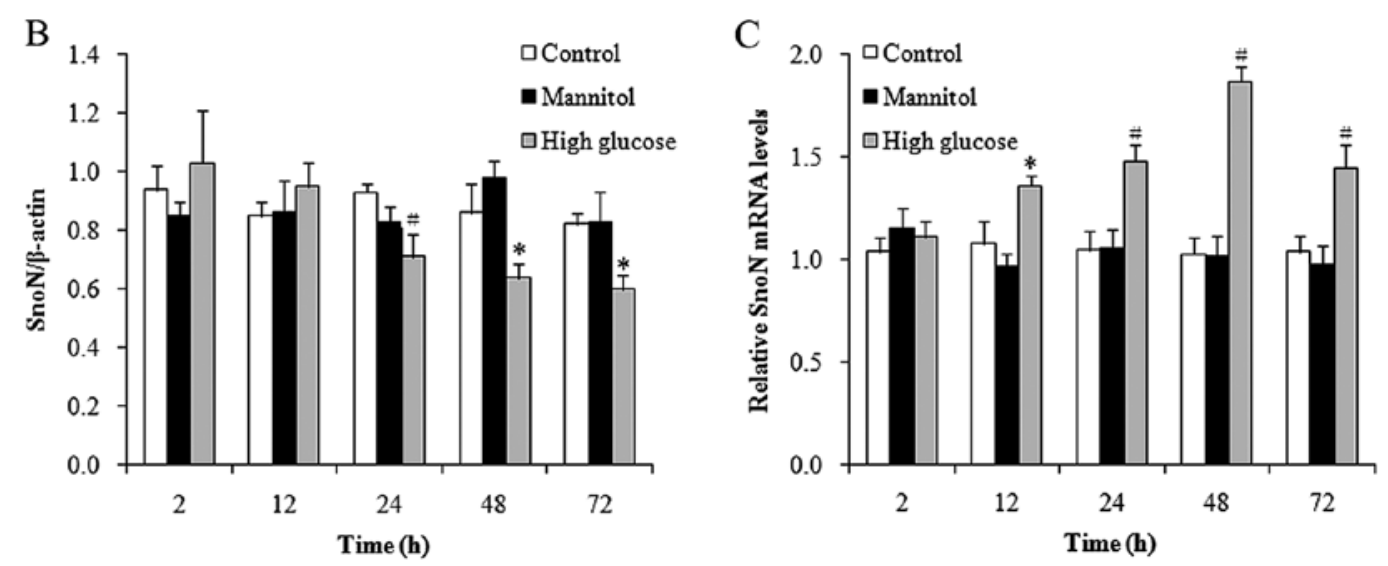

Figure 1. Ski-related novel protein $\mathrm{N}(\mathrm{SnoN})$ protein expression is downregulated while its mRNA expression is increased in primary human renal proximal tubule epithelial cells (hRPTECs) under high-glucose conditions. hRPTECs were incubated with normal glucose (control), $24.5 \mathrm{mmol} / 1 \mathrm{mannitol}$ (mannitol), or $30 \mathrm{mmol} / 1 \mathrm{D}$-glucose (high glucose) for various periods of time as indicated. (A) Western blot analysis shows the gradual reduction of SnoN protein in hRPTECs under high-glucose conditions. (B) Graphic representation of SnoN abundance under different conditions after normalization with $\beta$-actin. (C) SnoN mRNA expression in hRPTECs cultured under different conditions was detected by RT-qPCR. Data are expressed as the means \pm SD of 3 experiments. ${ }^{*} \mathrm{P}<0.05$ vs. control (normal glucose) at the same time point. ${ }^{\text {} P}<0.01$ vs. control at the same time point.

spectrophotometry. Complementary (c)DNA was synthesized from $1 \mu \mathrm{g}$ total RNA using oligo(dT) primers and the Reverse Transcription system (Promega Corp., Madison, WI, USA) at $42^{\circ} \mathrm{C}$ for $60 \mathrm{~min}$ as recommended by the manufacturer. qPCR with $1 \mu \mathrm{l}$ cDNA was performed using a TaqMan Gene Expression assay for each mRNA and TaqMan Gene Expression Master Mix (Applied Biosystems, Foster City, CA, USA) in a total of $20 \mu \mathrm{l} /$ reaction. The PCR conditions were as follows: $95^{\circ} \mathrm{C}$ for $10 \mathrm{~min}, 40$ cycles at $95^{\circ} \mathrm{C}$ for $15 \mathrm{sec}$, and $60^{\circ} \mathrm{C}$ for $60 \mathrm{sec}$ for amplification. All reactions were performed in triplicate. The results were analyzed using the $2^{-\Delta \Delta \mathrm{Ct}}$ method.

Co-immunoprecipitation. The hRPTECs were harvested after the cells were cultured in medium containing $30 \mathrm{mmol} / \mathrm{l}$ D-glucose for $24 \mathrm{~h}$. The cells were lysed and centrifuged at $12,000 \mathrm{x} \mathrm{g}$ for $20 \mathrm{~min}$ at $4^{\circ} \mathrm{C}$. The supernatants were collected for immunoprecipitation. After preclearing with normal host IgG (Santa Cruz Biotechnology, Inc.), the lysates were immunoprecipitated overnight at $4^{\circ} \mathrm{C}$ with anti-Smurf2 antibody (1/100 $\mu \mathrm{g}$ total protein), followed by precipitation with $20 \mu \mathrm{l}$ of protein A/G Plus-Agarose (Santa Cruz Biotechnology, Inc.) for $4 \mathrm{~h}$ at $4^{\circ} \mathrm{C}$. After 3 washes, the precipitated complexes were separated by $10 \%$ SDS-PAGE which was followed by western blot analysis.

Statistical analysis. All data are expressed as the means \pm SD. Statistical analysis of the data was performed using SPSS 17.0 software (SPSS, Inc., Chicago, IL, USA). Comparisons among the experimental groups were performed using one-way analysis of variance (ANOVA) followed by Scheffe's test. A value of $\mathrm{P}<0.05$ was considered to indicate a statistically significant difference.

\section{Results}

High-glucose conditions induce the downregulation of SnoN in hRPTECs at the post-transcriptional level. To determine whether high-glucose conditions decrease SnoN protein levels, we cultured the hRPTECs in media containing normal glucose $(5.5 \mathrm{mmol} / \mathrm{l}$ D-glucose), high glucose $(30 \mathrm{mmol} / \mathrm{l}$ D-glucose) or in medium of a high osmolarity $(5.5 \mathrm{mmol} / 1 \mathrm{D}$ -glucose $+24.5 \mathrm{mmol} / \mathrm{l} \mathrm{D}$-mannitol) for different periods of time and we measured the SnoN protein levels by western blot analysis. Compared with the cells cultured under normalglucose conditions, in the cells cultured under high-glucose conditions, SnoN protein expression was significantly downregulated after $24 \mathrm{~h}$ (Fig. 1A and B). This may not be due to the hypertonic pressure of $30 \mathrm{mmol} / 1 \mathrm{D}$-glucose, as $24.5 \mathrm{mmol} / 1$ mannitol, which has an equal osmotic pressure as $30 \mathrm{mmol} / \mathrm{l}$ D-glucose, did not apparently alter the abundance of SnoN protein (Fig. 1A and B). To determine whether the decrease in SnoN protein expression resulted from the downregulation of SnoN mRNA expression, we measured the SnoN mRNA levels by RT-qPCR. Surprisingly, the SnoN mRNA levels were significantly upregulated in the hRPTECs cultured under highglucose conditions from 12-72 $\mathrm{h}$ (Fig. 1C) in contrast to those of the cells grown in medium containing normal glucose and of high osmolarity. These results indicated that $\mathrm{SnoN}$ expression was reduced under high-glucose conditions in the hRPTECs at the post-transcriptional level.

Smurf2 expression is induced by high-glucose conditions in hRPTECs. Previous studies have shown that the E3 ubiquitin ligase, Smurf2, targets SnoN for proteasome-mediated degradation $(14,15)$. In this study, to determine whether Smurf2 


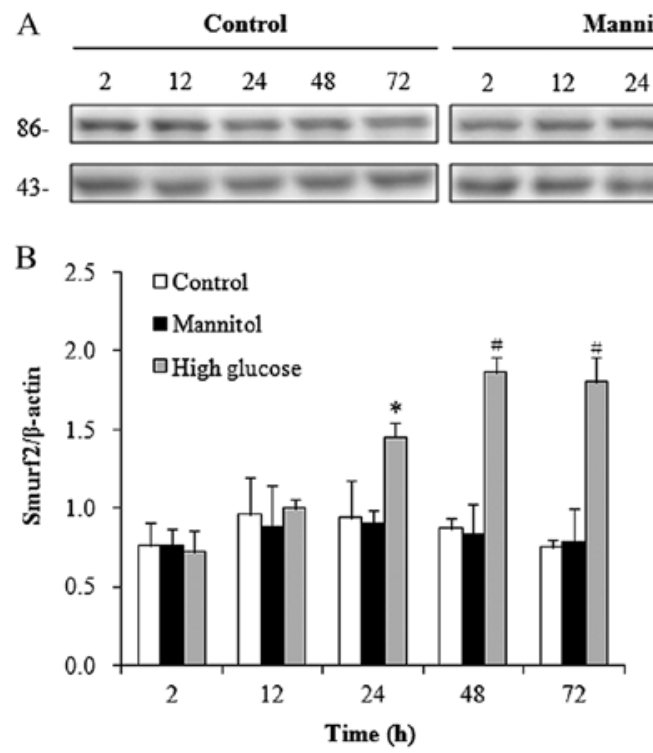

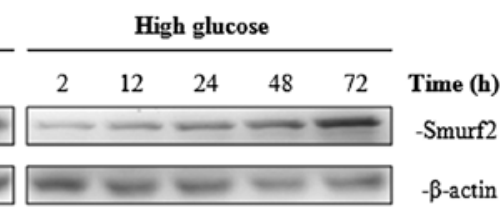

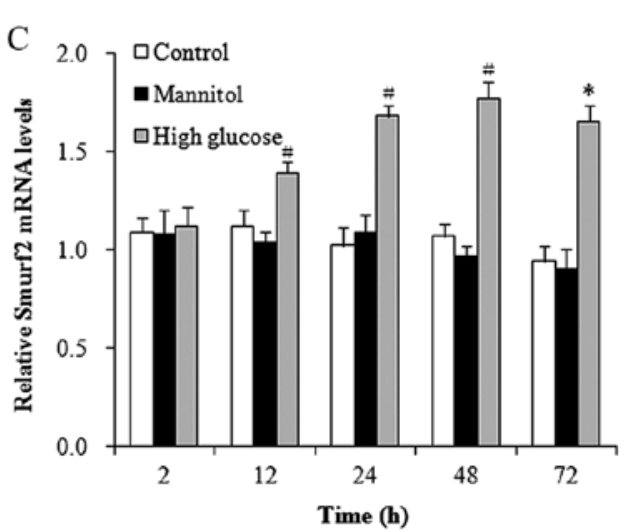

Figure 2. Smad ubiquitination regulatory factor-2 (Smurf2) protein and mRNA expression are induced in primary human renal proximal tubule epithelial cells (hRPTECs) under high-glucose conditions. (A) Western blot analysis shows the induction of Smurf2 protein in hRPTECs under high-glucose conditions in a time-dependent manner. (B) Graphic representation of the relative abundance of Smurf2 under different conditions after normalization with $\beta$-actin. (C) Smurf2 mRNA expression in hRPTECs under different conditions was determined by RT-qPCR. Data are expressed as the means \pm SD of 3 experiments. ${ }^{*} \mathrm{P}<0.05$ vs. control (normal glucose) at the same time point. ${ }^{\#} \mathrm{P}<0.01$ vs. control at the same time point.

contributes to the downregulation of SnoN under high-glucose conditions, we measured the protein expression levels of Smurf2 in the hRPTECs under high-glucose conditions. Western blot analysis revealed that Smurf2 expression was significantly induced from $24 \mathrm{~h}$ in the hRPTECs cultured under high-glucose conditions in a time-dependent manner (Fig. 2A and B). RT-qPCR demonstrated the increased mRNA expression of Smurf2 in the hRPTECs cultured under high-glucose conditions from $12 \mathrm{~h}$ (Fig. 2C). Similar to the results observed for SnoN, the hypertonic culture environment did not affect the neither protein expression of Smurf2 (Fig. 2A and B) nor its mRNA expression (Fig. 2C) in the hRPTECs. These results demonstrated that the high-glucose conditions enhanced the expression of Smurf2.

Knockdown of Smurf2 by siRNA stabilizes SnoN expression in hRPTECs under high-glucose conditions. Our above-mentioned observations suggest that the upregulation of Smurf2 may contribute to the downregulation of SnoN under high-glucose conditions. To further examine this hypothesis, we first used an RNA interference (RNAi) approach to knockdown Smurf2 expression in the hRPTECs under high-glucose conditions. To ensure the specificity of Smurf2 inhibition, two different doublestranded Smurf2 siRNAs and a control siRNA were separately transiently transfected into the hRPTECs. The transfection efficiency was observed by calculating the ratio of red fluorescent cells among the total cells. Approximately $70-80 \%$ of the hRPTECs transfected with siRNA expressed a red fluorescence signal in the nuclei, indicating efficient transfection (Fig. 3A). The Smurf 2 mRNA levels were reduced by $62 \%$ following transfection with Smurf2 siRNA-1 and by $45 \%$ following transfection with Smurf2 siRNA-2 compared with the cells transfected with the control siRNA (Fig. 3B). Similarly, the Smurf 2 protein levels were reduced by $50 \%$ following transfection with Smurf2 siRNA-1 and by $34 \%$ following transfection with Smurf2 siRNA-2 compared with the cells transfected with the control siRNA (Fig. 3C).

Subsequently, we examined the SnoN protein levels following the knockdown of Smurf2 expression in the hRPTECs under high-glucose conditions. The Smurf2 protein levels were increased while the SnoN protein levels were decreased when the cells were cultured in high-glucose medium (Fig. 4A and B). Following the knockdown of Smurf2 expression, the SnoN protein levels were increased by 1.5 -fold in the cells cultured in high-glucose medium, to similar levels observed under normal glucose conditions (Fig. 4B). These results suggest that high-glucose conditions downregulate SnoN expression by upregulating Smurf 2 in the hRPTECs.

Smurf2 physically interacts with SnoN under high-glucose conditions. To confirm the involvement of the ubiquitinproteasomal degradation pathway in the downregulation of SnoN expression under high-glucose conditions, we used the proteasome specific inhibitor, MG132, to block the ubiquitinproteasomal pathway in the hRPTECs. As shown in Fig. 5A, treatment with MG132 prevented the degradation of SnoN protein in the hRPTECs under high-glucose conditions. We then examined the potential physical interaction between Smurf2 and SnoN in the hRPTECs by protein co-immunoprecipitation. SnoN was detected in the immunocomplexes that were precipitated with Smurf2-specific antibody under high-glucose conditions, while it was undetectable when applied with control IgG or under normal glucose conditions (Fig. 5B). These results demonstrated that Smurf2 physically interacted with SnoN under high-glucose conditions and suggested that SnoN protein was subjected to proteasome-mediated degradation by Smurf2 in hRPTECs under high-glucose conditions.

Pharmacological inhibition of TGF- $\beta 1$ signaling with SB-431542 inhibits high-glucose-induced Smad2 activation and Smurf2 


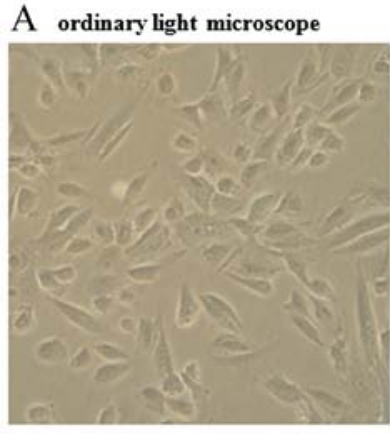

B

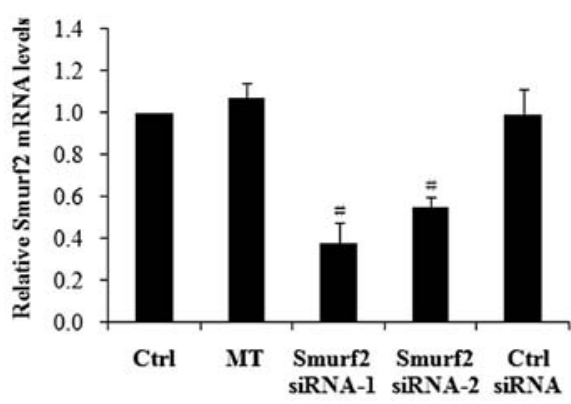

fluorescence microscope

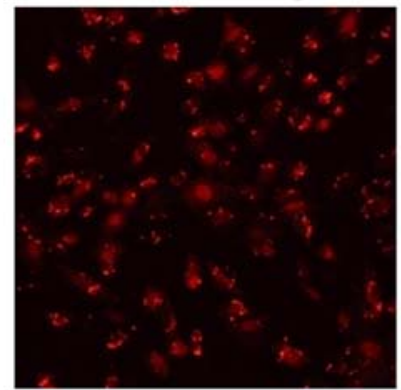

$\mathrm{C}$

86-

43-

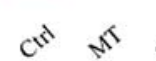

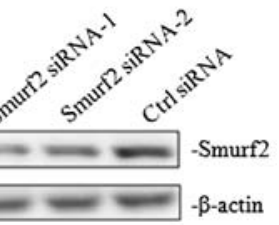

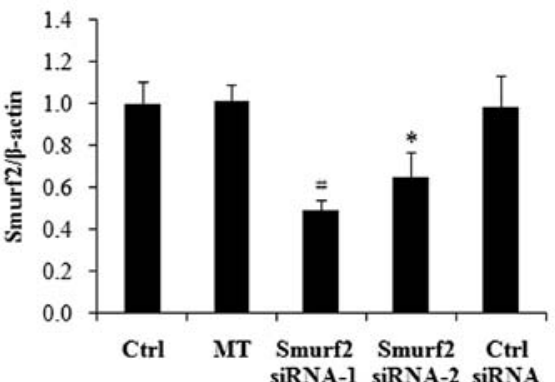

Figure 3. Smad ubiquitination regulatory factor-2 (Smurf2)-siRNA inhibits Smurf2 expression in primary human renal proximal tubule epithelial cells (hRPTECs). (A) An Alexa Fluor ${ }^{\circledR} 555$-labeled, double-stranded RNA (dsRNA) duplex was transfected into hRPTECs. Transfection efficiency was 70-80\% by visual inspection under a fluorescence microscope (x200 magnification). (B) Smurf2 mRNA expression was determined at $48 \mathrm{~h}$ following transfection of various siRNAs into hRPTECs by RT-qPCR. Ctrl, control; MT, mock transfection. (C) Western blot analysis of Smurf2 protein levels at $48 \mathrm{~h}$ following transfection of various siRNAs into hRPTECs. The bottom panel is a graphical representation of the relative Smurf2 levels after normalization with $\beta$-actin. Data relative to the control group (normalized to 1.0) are expressed as the means $\pm \mathrm{SD}$ of 3 experiments. ${ }^{\prime} \mathrm{P}<0.05$ versus control siRNA group. ${ }^{\sharp} \mathrm{P}<0.01$ versus control siRNA group.
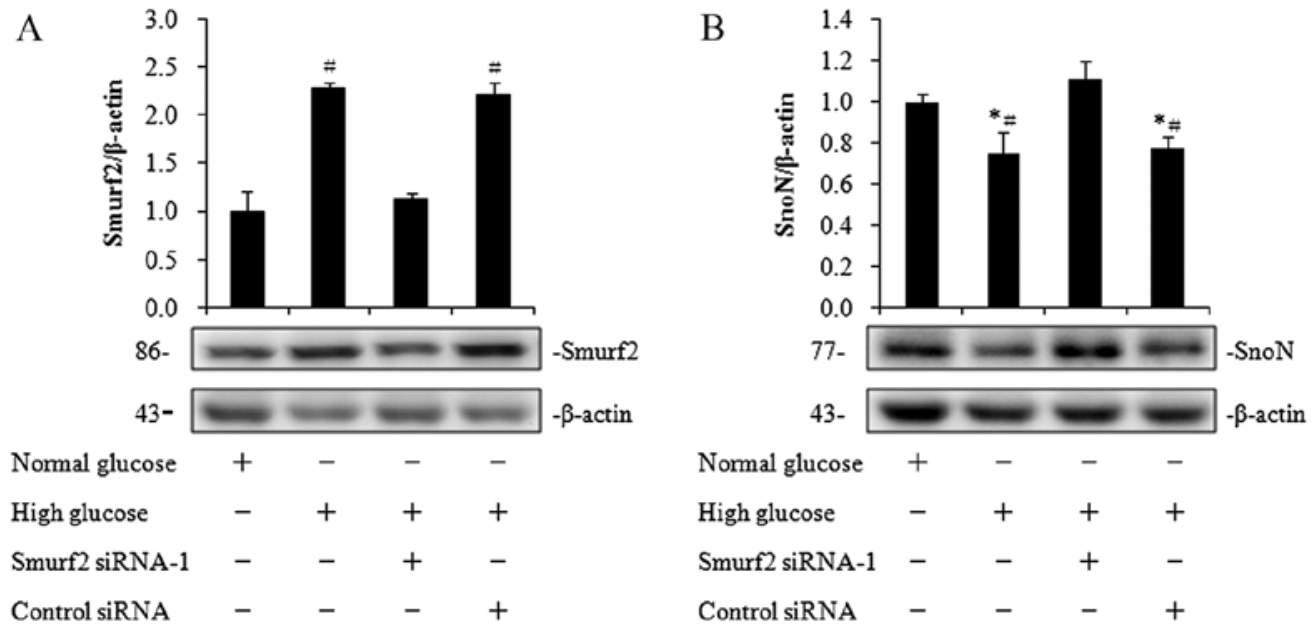

Figure 4. Knockdown of Smad ubiquitination regulatory factor-2 (Smurf2) expression increases Ski-related novel protein N (SnoN) protein expression in primary human renal proximal tubule epithelial cells (hRPTECs) under high-glucose conditions. hRPTECs were transiently transfected with control siRNA or Smurf2 siRNA-1 for $6 \mathrm{~h}$, and then the cells were cultured in high-glucose medium for an additional $48 \mathrm{~h}$. (A) Western blot analysis shows the expression levels of Smurf2 at $48 \mathrm{~h}$ after transfection. Graphical representation of the relative Smurf2 levels is shown in the upper panel after normalization with $\beta$-actin. (B) Western blot analysis of SnoN expression levels at $48 \mathrm{~h}$ following transfection. Graphical representation of the relative abundance of SnoN protein is shown in the upper panel after normalization with $\beta$-actin. Data relative to the normal glucose group (normalized to 1.0 ) are expressed as the means \pm SD of 3 experiments. "P<0.05 vs. the normal glucose group. ${ }^{*} \mathrm{P}<0.01$ vs. Smurf2 siRNA-1 group.

expression. It has been well documented that TGF- $\beta 1$ plays a key role in the pathophysiology of DN and that high glucose activates TGF- $\beta 1$ signaling $(4,10)$. Thus, to determine whether the upregulation of Smurf 2 results from enhanced TGF- $\beta 1$ signaling in the hRPTECs under high-glucose conditions, we treated the hRPTECs under high-glucose conditions with the TGF- $\beta$ type I receptor specific inhibitor, SB-431542, and examined the expression of Smurf2. The phosphorylation of Smad2, a downstream 


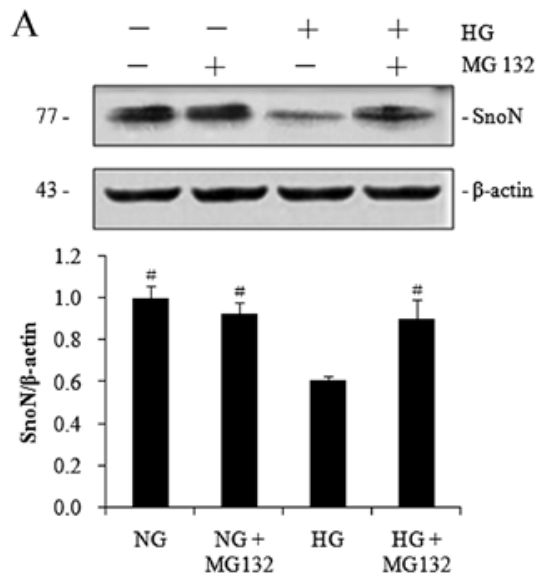

B

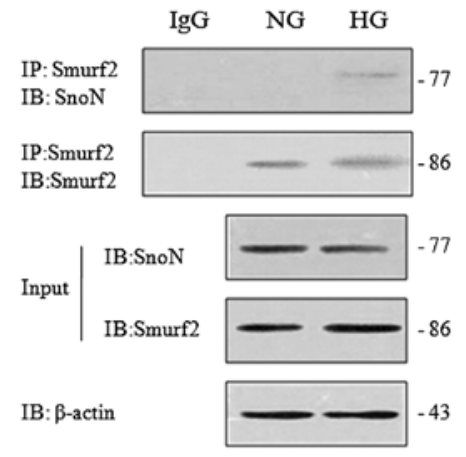

Figure 5. MG132 attenuates high-glucose-induced degradation of Ski-related novel protein N (SnoN) protein, and Smad ubiquitination regulatory factor-2 (Smurf2) physically interacted with SnoN in primary human renal proximal tubule epithelial cells (hRPTECs) cultured in high-glucose (HG) medium. (A) Western blot analysis shows that the proteasome inhibitor, MG132, partially abolished high-glucose-induced degradation of SnoN protein. The bottom panel is a graphical representation of relative SnoN levels after normalization with $\beta$-actin. Data relative to the normal glucose (NG) group (normalized to 1.0) are expressed as the means \pm SD of 3 experiments. ${ }^{~} \mathrm{P}<0.01$ vs. HG group. (B) Smurf2 physically interacted with SnoN in hRPTECs cultured in medium containing $30 \mathrm{mmol} / 1$ D-glucose for $24 \mathrm{~h}$. Cell lysates were immunoprecipitated with a specific antibody against Smurf2, followed by western blot analysis (immunoblotting) with anti-SnoN or anti-Smurf2 antibodies, respectively.

A
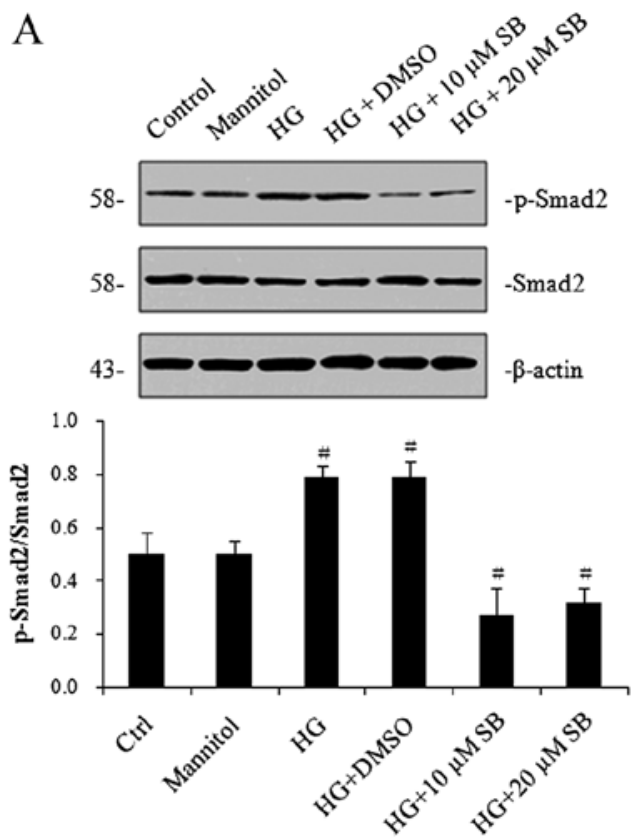

B
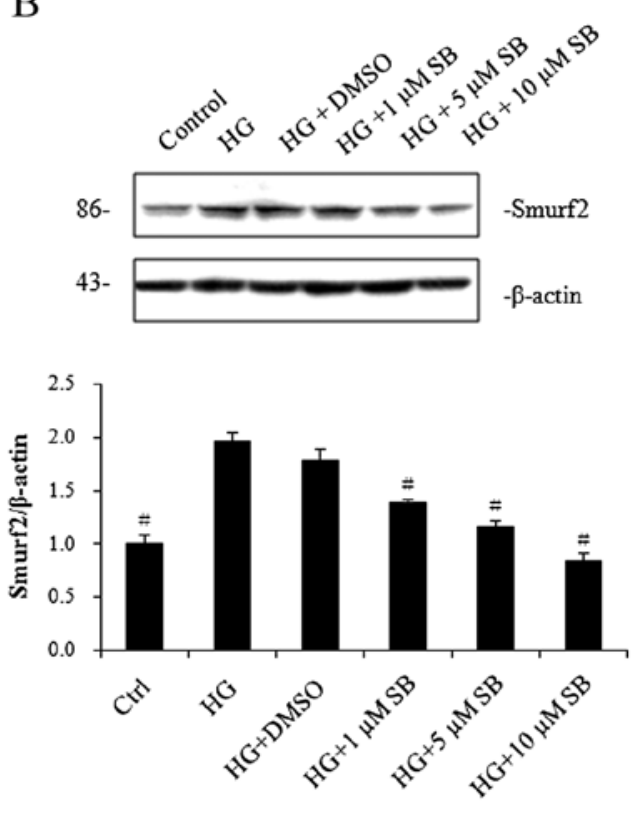

Figure 6. The upregulation of Smad ubiquitination regulatory factor-2 (Smurf2) protein by high glucose is inhibited by SB-431542 in human renal proximal tubule epithelial cells (hRPTECs). The cells were treated with various concentrations of SB-431542 or DMSO as indicated for $48 \mathrm{~h}$ in medium containing $30 \mathrm{mmol} / 1$ D-glucose. (A) SB-431542 blocks the phosphorylation of Smad2 in hRPTECs. Western blot analysis showed activation of Smad2 in hRPTECs under high-glucose conditions, and this activation was prevented by SB-431542. Graphical representation of the phosphorylated (p-)Smad2/Smad2 levels is shown in the bottom panel. Data are expressed as the means $\pm \mathrm{SD}$ of 3 experiments (data of the control group were not normalized to 1.0 ). ${ }^{\text {P }}<0.01 \mathrm{vs}$. control group. SB, SB-431542; HG, high glucose. (B) Western blot analysis demonstrated that the expression of Smurf 2 protein is suppressed by SB-431542 in a dose-dependent manner. The bottom panel is a graphical representation of relative Smurf 2 levels after normalization with $\beta$-actin. Data are expressed as the means $\pm \mathrm{SD}$ of 3 experiments. ${ }^{\sharp} \mathrm{P}<0.01 \mathrm{vs}$. HG group.

target of TGF- $\beta 1$ signaling, was stimulated by high glucose and prevented by SB-431542 (Fig. 6A), supporting the conclusion that high glucose activates TGF- $\beta 1$ signaling (4). Intriguingly, the high-glucose induced increase in the protein levels of Smurf2 was significantly inhibited by SB-431542 in a dose-dependent manner (Fig. 6B). These results demonstrated that SB-431542 blocked the high-glucose-induced activation of Smad2 and inhibited the protein expression of the Smurf2.

\section{Discussion}

In this study, we found that high-glucose conditions decreased the protein level, while increasing the mRNA level of SnoN in the hRPTECs, and concurrently upregulated Smurf2. Moreover, Smurf2 physically interacted with SnoN under high-glucose conditions and the knockdown of Smurf2 by siRNA or MG132 abolished the downregulation of SnoN under 
high-glucose conditions. Furthermore, the pharmacological inhibition of TGF- $\beta 1$ signaling with SB-431542 inhibited the high-glucose-induced Smad2 activation and Smurf2 expression. Our findings suggest that the downregulation of SnoN expression in hRPTECs under high-glucose conditions is mediated by an increase in Smurf2 expression through TGF- $\beta 1 /$ Smad signaling.

SnoN is a negative regulator of TGF- $\beta$ signaling as a consequence of binding to Smad proteins $(8,9)$. SnoN disrupts the formation of a functional complex between $\mathrm{Smad} 4$ and activated Smad2/3, thereby blocking the Smad complexes from activating the transcription of TGF- $\beta 1$ target genes $(16,17)$. Furthermore, SnoN may recruit other transcriptional co-repressors, such as the nuclear hormone receptor co-repressor $(\mathrm{N}-\mathrm{CoR})$, and prevent the binding of Smads to the transcriptional co-activator, p300/ CREB-binding protein (CBP) $(6,8,17)$. These mechanisms may operate together to suppress TGF- $\beta 1 / \mathrm{Smad}$ signaling through SnoN. On the other hand, Yang et al (18) demonstrated that the threshold for the TGF- $\beta 1$ response was reduced significantly by the complete depletion of SnoN and that a minimal amount of TGF- $\beta 1$ was sufficient to trigger a full-scale TGF- $\beta 1$ response under chronic disease conditions. It is widely accepted that hyperactive TGF- $\beta 1$ signaling plays a crucial role in the genesis and progression of diabetic renal injuries $(11,12,19)$. Thus, it is possible that the downregulation of SnoN protein expressino under high-glucose conditions may lead to hyperactive TGF- $\beta 1 /$ Smad signaling and promote the pathogenesis of DN.

However, the mechanisms that regulate the expression of SnoN are not yet fully understood. SnoN transcription is strongly induced by TGF- $\beta$ itself (6). In this study, we demonstrated that the mRNA expression levels of SnoN were significantly increased in the hRPTECs under high-glucose conditions, through an unknown mechanism. This upregulation of SnoN transcription may function as a negative feedback mechanism to inhibit TGF- $\beta 1$ signaling. Although SnoN mRNA levels were significantly increased, the SnoN protein levels were decreased under these conditions, suggesting that SnoN is mainly regulated at the post-transcriptional level.

Intracellular protein degradation is a tightly regulated process that is essential to sustain normal cellular functions and is mainly controlled by the ubiquitin-proteasome system, which contains an E1 ubiquitin-activating enzyme, an E2 ubiquitin-conjugating enzyme, and a substrate specific E3 ubiquitin ligase. The E3 ubiquitin ligase defines the substrate selectivity and the subsequent degradation by the $26 \mathrm{~S}$ proteasome $(20,21)$. As previously demonstrated, the deregulation of the ubiquitinproteasome system disrupts normal cellular homeostasis and leads to the development of a number of human diseases, including Liddle syndrome, ischemic acute renal failure and obstructive nephropathy $(14,15,20)$. Smurf2, homologous to the E6-AP carboxyl terminus (HECT) domain-containing E3 ubiquitin ligase, targets SnoN for ubiquitin-mediated degradation by the proteasomes (22). In the present study, we demonstrated that SnoN reduction in the hRPTECs, cultured under high-glucose conditions, was mediated by enhanced proteasome-dependent degradation. Firstly, SnoN protein levels were decreased in spite of an increase in its mRNA expression. Secondly, Smurf2 mRNA and protein levels were induced in the hRPTECs cultured in high-glucose medium.
Thirdly, the knockdown of Smurf2 expression by siRNA stabilized the SnoN protein levels in the hRPTECs. Finally, Smurf2 physically interacted with SnoN, and treatment with MG132 restored SnoN expression. Thus, Smurf2 targeting of SnoN for degradation by the ubiquitin-proteasome system is one of the mechanisms responsible for the downregulation of SnoN in hRPTECs under high-glucose conditions. However, other E3 ubiquitin ligases, such as the anaphase-promoting complex and Arkadia, have also been shown to target SnoN for degradation in response to TGF- $\beta 1(23,24)$. Whether they are also involved in the proteasome-dependent degradation of SnoN under high-glucose conditions remains to be determined.

The mRNA expression of Smurf2 is rapidly induced by TGF- $\beta$ itself $(25,26)$. Furthermore, Smurf 2 induction by TGF- $\beta 1$ requires Smad signaling rather than the p38 mitogen-activated protein kinase (MARK), phosphoinositide 3-kinase (PI3K), c-Jun N-terminal kinase (JNK), or MEK signaling pathways (26). SB-431542 is a potent and specific inhibitor of the TGF- $\beta$ type I receptor $(27,28)$. Inman et al $(28)$ reported that SB-431542 inhibited the TGF- $\beta$-induced phosphorylation of Smad 2 and blocked the activation of TGF- $\beta$ signaling. In this study, we found that SB-431542 suppressed the expression of phosphorylated (p-)Smad2 and Smurf2 in a dose-dependent manner. These observations suggest that the upregulation of Smurf 2 by TGF- $\beta /$ Smad signaling contributes to the degradation of SnoN under high-glucose conditions.

In conclusion, in this study, we demonstrated that the downregulation of SnoN expression in hRPTECs under high-glucose conditions is mediated by an incresae in Smurf2 expression through the TGF- $\beta 1 /$ Smad signaling pathway. Our findings suggest that a decrease in SnoN expression may eliminate the negative regulatory mechanism for TGF- $\beta 1$ signaling and amplify profibrotic TGF- $\beta 1$ signaling, leading to a vicious cycle between Smurf2 expression and TGF- $\beta 1$ action. The preservation of SnoN by a proteasome inhibitor or by the knockdown of Smurf2, may be an effective approach for confining pathological TGF- $\beta 1$ activity.

\section{Acknowledgements}

The present study was supported by grants from the National Natural Science Foundation of China (no. 81300607) and the Beijing Municipal Science and Technology Commission Fund (no. D131100004713001).

\section{References}

1. Tang SC and Lai KN: The pathogenic role of the renal proximal tubular cell in diabetic nephropathy. Nephrol Dial Transplant 27: 3049-3056, 2012.

2. Phillips AO and Steadman R: Diabetic nephropathy: the central role of renal proximal tubular cells in tubulointerstitial injury. Histol Histopathol 17: 247-252, 2002.

3. Vallon V and Thomson SC: Renal function in diabetic disease models: the tubular system in the pathophysiology of the diabetic kidney. Annu Rev Physiol 74: 351-375, 2012.

4. Loeffler I and Wolf G: Transforming growth factor- $\beta$ and the progression of renal disease. Nephrol Dial Transplant 29 (Suppl 1): i37-i45, 2014.

5. Massagué $\mathbf{J}$ and Gomis RR: The logic of TGFbeta signaling. FEBS Lett 580: 2811-2820, 2006.

6. Stroschein SL, Wang W, ZhouS, Zhou Q and Luo K: Negative feedback regulation of TGF-beta signaling by the SnoN oncoprotein. Science 286: 771-774, 1999. 
7. Wotton D and Massagué J: Smad transcriptional corepressors in TGF beta family signaling. Curr Top Microbiol Immunol 254 145-164, 2001.

8. Luo K: Ski and SnoN: Negative regulators of TGF-beta signaling Curr Opin Genet Dev 14: 65-70, 2004.

9. Deheuninck J and Luo K: Ski and SnoN, potent negative regulators of TGF-beta signaling. Cell Res 19: 47-57, 2009.

10. Chen S, Jim B and Ziyadeh FN: Diabetic nephropathy and transforming growth factor-beta:transforming our view of glomerulosclerosis and fibrosis build-up. Semin Nephrol 23 . 532-543, 2003.

11. Hills CE and Squires PE: The role of TGF- $\beta$ and epithelial-to mesenchymal transition in diabetic nephropathy. Cytokine Growth Factor Rev 22: 131-139, 2011.

12. Lan HY: Transforming growth factor- $\beta /$ Smad signalling in diabetic nephropathy. Clin Exp Pharmacol Physiol 39: 731-738, 2012.

13. Liu R, Wang Y, Xiao Y, Shi M, Zhang G and Guo B: SnoN as a key regulator of the high glucose-induced epithelial-mesenchymal transition in cells of the proximal tubule. Kidney Blood Press Res 35: 517-528, 2012.

14. Tan R, Zhang J, Tan X, Zhang X, Yang J and Liu Y: Downregulation of SnoN expression in obstructive nephropathy is mediated by an enhanced ubiquitin-dependent degradation. J Am Soc Nephrol 17: 2781-2791, 2006.

15. Fukasawa H, Yamamoto T, Togawa A, Ohashi N, Fujigaki Y, Oda T, Uchida C, Kitagawa K, Hattori T, Suzuki S, et al: Ubiquitin-dependent degradation of SnoN and Ski is increased in renal fibrosis induced by obstructive injury. Kidney Int 69: 1733-1740, 2006

16. Liu X, Sun Y, Weinberg RA and Lodish HF: Ski/Sno and TGF-beta signaling. Cytokine Growth Factor Rev 12: 1-8, 2001.

17. Wu JW, Krawitz AR, Chai J, Li W, Zhang F, Luo K and Shi Y: Structural mechanism of Smad4 recognition by the nuclear oncoprotein Ski: insights on Ski-mediated repression of TGF-beta signaling. Cell 111: 357-367, 2002.

18. Yang J, Zhang X, Li Y and Liu Y: Downregulation of Smad transcriptional corepressors SnoN and Ski in the fibrotic kidney: an amplification mechanism for TGF-betal signaling. J Am Soc Nephrol 14: 3167-3177, 2003
19. Ziyadeh FN: Mediators of diabetic renal disease: The case for TGF- $\beta$ as the major mediator. J Am Soc Nephrol 15 (Suppl 1): S55-S57, 2004.

20. Debigaré R and Price SR: Proteolysis, the ubiquitin-proteasome system, and renal diseases. Am J Physiol Renal Physiol 285: F1-F8, 2003.

21. Mani A and Gelmann EP: The ubiquitin-proteasome pathway and its role in cancer. J Clin Oncol 23: 4776-4789, 2005.

22. Bonni S, Wang HR, Causing CG, Kavsak P, Stroschein SL, Luo K and Wrana JL: TGF-beta induces assembly of a Smad2-Smurf2 ubiquitin ligase complex that targets SnoN for degradation. Nat Cell Biol 3: 587-595, 2001.

23. Wan Y, Liu X and Kirschner MW: The anaphase-promoting complex mediates TGF-beta signaling by targeting $\mathrm{SnoN}$ for destruction. Mol Cell 8: 1027-1039, 2001.

24. Nagano Y, Mavrakis KJ, Lee KL, Fujii T, Koinuma D, Sase H, Yuki K, Isogaya K, Saitoh M, Imamura T, et al: Arkadia induces degradation of SnoN and c-Ski to enhance transforming growth factor-beta signaling. J Biol Chem 282: 20492-20501, 2007.

25. Ohashi N, Yamamoto T, Uchida C, Togawa A, Fukasawa H, Fujigaki Y, Suzuki S, Kitagawa K, Hattori T, Oda T, et al: Transcriptional induction of Smurf2 ubiquitin ligase by TGF-beta. FEBS Lett 579: 2557-2563, 2005.

26. Tan R, He W, Lin X, Kiss LP and Liu Y: Smad ubiquitination regulatory factor-2 in the fibrotic kidney: Regulation, target specificity, and functional implication. Am J Physiol Renal Physiol 294: F1076-F1083, 2008.

27. Callahan JF, Burgess JL, Fornwald JA, Gaster LM, Harling JD, Harrington FP, Heer J, Kwon C, Lehr R, Mathur A, et al: Identification of novel inhibitors of the transforming growth factor betal (TGF- $\beta 1$ ) type 1 receptor (ALK5). J Med Chem 45: 999-1001, 2002.

28. Inman GJ, Nicolás FJ, Callahan JF, Harling JD, Gaster LM, Reith AD, Laping NJ and Hill CS: SB-431542 is a potent and specific inhibitor of transforming growth factor-beta superfamily type I activin receptor-like kinase (ALK) receptors ALK4, ALK5, and ALK7. Mol Pharmacol 62: 65-74, 2002. 